\title{
Homogeneously high expression of CD32b makes it a potential target for CAR-T therapy for chronic lymphocytic leukemia
}

\author{
Guoling Wang ${ }^{1,2+}$, Xiaolei Sun ${ }^{1 \dagger}$, Shiyu Zuo ${ }^{1}$, Chuo Li', Qing Niu' ${ }^{1}$, Yonghui Xia', Yuan Meng ${ }^{1}$, Min Liu', \\ Zihao Fang ${ }^{3}$, Xi Yang ${ }^{3}$, Yanyu Jiang ${ }^{4}$, Sheng Wang ${ }^{5}$, Haidong Cui ${ }^{6}$, Huifang Huang ${ }^{7}$, Erlie Jiang ${ }^{1}$, \\ Dongming Zhou ${ }^{8}$, Qi Deng ${ }^{4^{*}}$, Jing Pan ${ }^{9^{*}}$ and Xiaoming Feng ${ }^{1,2,7^{*}}$ (i)
}

\begin{abstract}
CD19 chimeric antigen receptor (CAR)-T cells have been used to treat patients with refractory chronic lymphocytic leukemia (CLL). However, approximately 50\% of patients do not respond to this therapy. To improve the clinical outcome of these patients, it is necessary to develop strategies with other optimal targets to enable secondary or combinational CAR-T cell therapy. By screening a panel of surface antigens, we found that CD32b (FcyRllb) was homogeneously expressed at high site density on tumor cells from CLL patients. We then developed a second-generation CAR construct targeting CD32b, and T cells transduced with the CD32 CAR efficiently eliminated the CD32 $\mathrm{b}^{+}$Raji leukemic cell line in vitro and in a mouse xenograft model. Furthermore, CD32b CAR-T cells showed cytotoxicity against primary human CLL cells that were cultured in vitro or transplanted into immunodeficient mice. The efficacy of CD32b CART cells correlated with the CD32b density on CLL cells. CD32b is not significantly expressed by non-B hematopoietic cells. Our study thus identifies CD32b as a potential target of CAR-T cell therapy for CLL, although further modification of the CAR construct with a safety mechanism may be required to minimize off-target toxicity.
\end{abstract}

Keywords: Chronic lymphocytic leukemia, CD32b, Chimeric antigen receptor, Antigen site density

\section{To the editor:}

Chronic lymphocytic leukemia (CLL) is a hematological neoplasm mostly diagnosed in the elderly. Refractory and relapsed $(\mathrm{r} / \mathrm{r})$ CLL patients have a poor prognosis

*Correspondence: kachydeng@126.com; panj@gobroadhealthcare.com; fengxiaoming@ihcams.ac.cn

${ }^{\dagger}$ Guoling Wang and Xiaolei Sun contributed equally to this work

1 State Key Laboratory of Experimental Hematology, National Clinical Research Center for Blood Diseases, Institute of Hematology and Blood Diseases Hospital, Chinese Academy of Medical Sciences and Peking Union Medical College, Tianjin 300020, China

${ }^{4}$ Department of Hematology, Tianjin First Central Hospital, School

of Medicine, Nankai University, Tianjin 300192, China

${ }^{9}$ State Key Laboratory of Experimental Hematology, Boren Clinical

Translational Center, Department of Hematology, Beijing Boren Hospital, Beijing 100070, China

Full list of author information is available at the end of the article with limited therapeutic options [1, 2]. Chimeric antigen receptor (CAR)-T cells targeting CD19 have shown activity in CLL, but can only induce complete remission in about $30 \%-60 \%$ of the patients $[3,4]$. It is essential to develop alternative targets for secondary or combinational CAR-T cell therapies for CLL.

Since target antigen site density and expression percentage on tumor cells are critical determinants of CAR-T cell efficacy [5], we aimed to identify a target antigen that was expressed at high levels on all CLL cells. The expression levels of B cell-associated antigens (CD19/ CD20/CD22/CD32) and 3 previously suggested targets $(\mathrm{CD} 23 / \mathrm{ROR} 1 / \mathrm{F} \mathrm{C} \mathrm{R})$ were examined on leukemic cells from CLL patients (Additional file 1: Table S1). CD32 (FCGR2) was expressed on $100 \% \mathrm{CD}^{+} \mathrm{CD} 19^{+} \mathrm{CLL}$ cells from all patients, similar to CD19 (Fig. 1a, Additional original author(s) and the source, provide a link to the Creative Commons licence, and indicate if changes were made. The images or other third party material in this article are included in the article's Creative Commons licence, unless indicated otherwise in a credit line to the material. If material is not included in the article's Creative Commons licence and your intended use is not permitted by statutory regulation or exceeds the permitted use, you will need to obtain permission directly from the copyright holder. To view a copy of this licence, visit http://creativecommons.org/licenses/by/4.0/. The Creative Commons Public Domain Dedication waiver (http://creativeco mmons.org/publicdomain/zero/1.0/) applies to the data made available in this article, unless otherwise stated in a credit line to the data. 
file 3: Fig. S1a). The average site density of CD32 was much higher than that of CD19 and the other antigens tested (Fig. 1b, Additional file 3: Fig. S1b). CD32 has three isoforms, CD32a, b, c; CD32b shares the same extracellular domain with CD32c [6]. RNA sequencing revealed that leukemic cells and Raji cells expressed abundant CD32b and low levels of CD32c but little CD32a (Fig. 1c). A soluble scFv derived from the CD32b-specific antibody 2B6 confirmed the homogeneously high expression of CD32b on CLL (Figs. 1d-f, Additional file 3: Fig. S1d). CD32b was not significantly expressed on hematopoietic stem/progenitor cells and most mature blood cells, but was expressed in a small proportion of dendritic cells (Fig. 1g-h).

Second-generation CAR constructs with scFv derived from the CD32b-specific antibodies 2B6 and NOV2108 were developed (Fig. 2a, b, Additional file 2). Since the CLL cell line MEC1 only partially expressed CD32, we used the Raji cell line, which had homogeneous CD32b expression, to evaluate the activity of CD32b CAR-T cells (Additional file 3: Fig. S2a, b). 2B6bbz showed slightly higher cytotoxicity against Raji cells than did 2108bbz in vitro, and $2 \mathrm{~B} 6 \mathrm{bbz} \mathrm{T}$ cells proliferated and strongly diminished the leukemia burden and prolonged survival in Raji-engrafted mice (Fig. 2c-i, Additional file 3: Fig. S2c, d).

In vitro cytotoxicity of $2 \mathrm{~B} 6 \mathrm{bbz}$ to primary CLL cells was higher than that of $2108 \mathrm{bbz}$ (Additional file 3: Fig. S3b). 2B6bbz $\mathrm{T}$ cells displayed similar anti-CLL cytotoxicity with CD19 CAR-T cells when the expression of CD19 and CD32 in leukemia was similar, and 2B6bbz was superior to CD19 CAR-T cells when the expression of CD32 in leukemia was higher than CD19 (Fig. 2j). Moreover, cytotoxicity of $2 \mathrm{~B} 6 \mathrm{bbz} \mathrm{T}$ cells positively correlated with CD32 density across different samples (Fig. 2k).

The in vivo anti-CLL activity of $2 \mathrm{~B} 6 \mathrm{bbz} \mathrm{T}$ cells was assessed in NSG mice transplanted with patient samples (Fig. 2l). 2B6bbz $\mathrm{T}$ cells were as potent as CD19 CAR-T cells: they achieved complete clearance of CLL in 80\% (8/10) of mice and showed robust proliferation in most mice (Fig. 2m-n, Additional file 3: Fig. S4b, c). Loss of CD32b expression was not observed (Additional file 3: Fig. S4d). Due to the limited persistence of CLL in mice, we could not evaluate whether $2 \mathrm{~B} 6 \mathrm{bbz} \mathrm{T}$ cells could provide a long-term cure effect. Since this model has been widely used to evaluate the in vivo efficacy of new drugs in CLL [7], our results indicate that CD32b CAR-T cells have potent cytotoxicity against CLL cells in vivo.

CD32b CAR-T cells may cause B cell aplasia, which can be managed with immunoglobulin infusion. Previous reports have shown the expression of CD32b in some normal tissues and cells, including airway smooth muscle cells, liver sinusoidal endothelial cells, Kupffer cells and placenta $[8,9]$, which may cause potential offtarget toxicities of CD32b CAR-T cell therapy. However, CD32b may still be an applicable target, since the potential off-target toxicity could be alleviated by decreasing CAR affinity for antigen or adopting a synNotch or zipper safety gate, which has been validated in various CAR-T cell studies [10-12]. Therefore, it would be feasible to improve the safety of CD32b CAR-T cells based on these modifications.

In summary, our study identifies CD32b as an antigen that is homogeneously expressed at high levels on CLL cells. CD32b CAR-T cells showed killing efficacy against primary CLL cells in vitro and in vivo. CD32b is therefore a promising target for CAR therapy in CLL, although further evaluation of off-target toxicities and optimization with safety modifications are needed before conducting clinical trials.

\footnotetext{
(See figure on next page.)

Fig. 1 CD32b is homogeneously expressed at high level on primary CLL cells, but not significantly expressed on non-B hematopoietic cells. a Expression (\% positive) of CD32 ( $n=41), C D 19(n=41), C D 20(n=33), C D 22(n=29), C D 23(n=29), R O R 1(n=22)$ and Fc $\mu R(n=22)$ in CLL samples (from CLL patients in Additional file 1: Table S1). $\mathbf{b}$ Evaluation for site density of CD32 and other antigens in CLL patients (sample size was the same as a) using Quantibrite-PE beads. c Transcriptional profile of Fcgr2a, Fcgr2b and Fcgr2c from 2 CLL samples and Raji cell line by RNA sequencing. d Flow cytometric analysis of surface expression of CD32, CD32b and CD19 in 7 CLL patients. e Expression (\% positive) of CD32, CD32b and CD19 in CLL patients $(n=7)$. f Site density comparison among CD32b, CD32 and CD19 in CLL patients $(n=7)$. Data in e-f belong to Pt 42-48, and the expression of CD32 and CD19 on samples from Pt 42-48 is not included in $\mathbf{a}-\mathbf{b}$. $\mathbf{h}$ Flow cytometric analysis of surface expression of CD32 on peripheral blood cells and HSPCs (CD34 ${ }^{+} \mathrm{CD} 38^{-} \mathrm{HSCs}$ and $\left.\mathrm{CD} 34^{+} \mathrm{CD} 38^{+} \mathrm{HPCs}\right)$ from a healthy donor. i Flow cytometric analysis of $\mathrm{CD} 32 \mathrm{~b}$ expression on normal peripheral blood cells and HSPCs from a healthy donor. FPKM: expected number of Fragments Per Kilobase of transcript sequence per Millions base pairs sequenced. HSC, hematopoietic stem cell; HPC, hematopoietic progenitor cell; NK, natural killer; DC, dendritic cells. Data were representative of two independent experiments. Unpaired two-tailed Student's t test was used for statistical analyses in $\mathbf{a}, \mathbf{b} ;$ paired two-tailed Student's t test was used in $\mathbf{e}$ and $\mathbf{f}\left({ }^{*} P<0.05\right.$, ${ }^{* *} P<0.01$, $\left.{ }^{* * *} P<0.001\right)$
} 

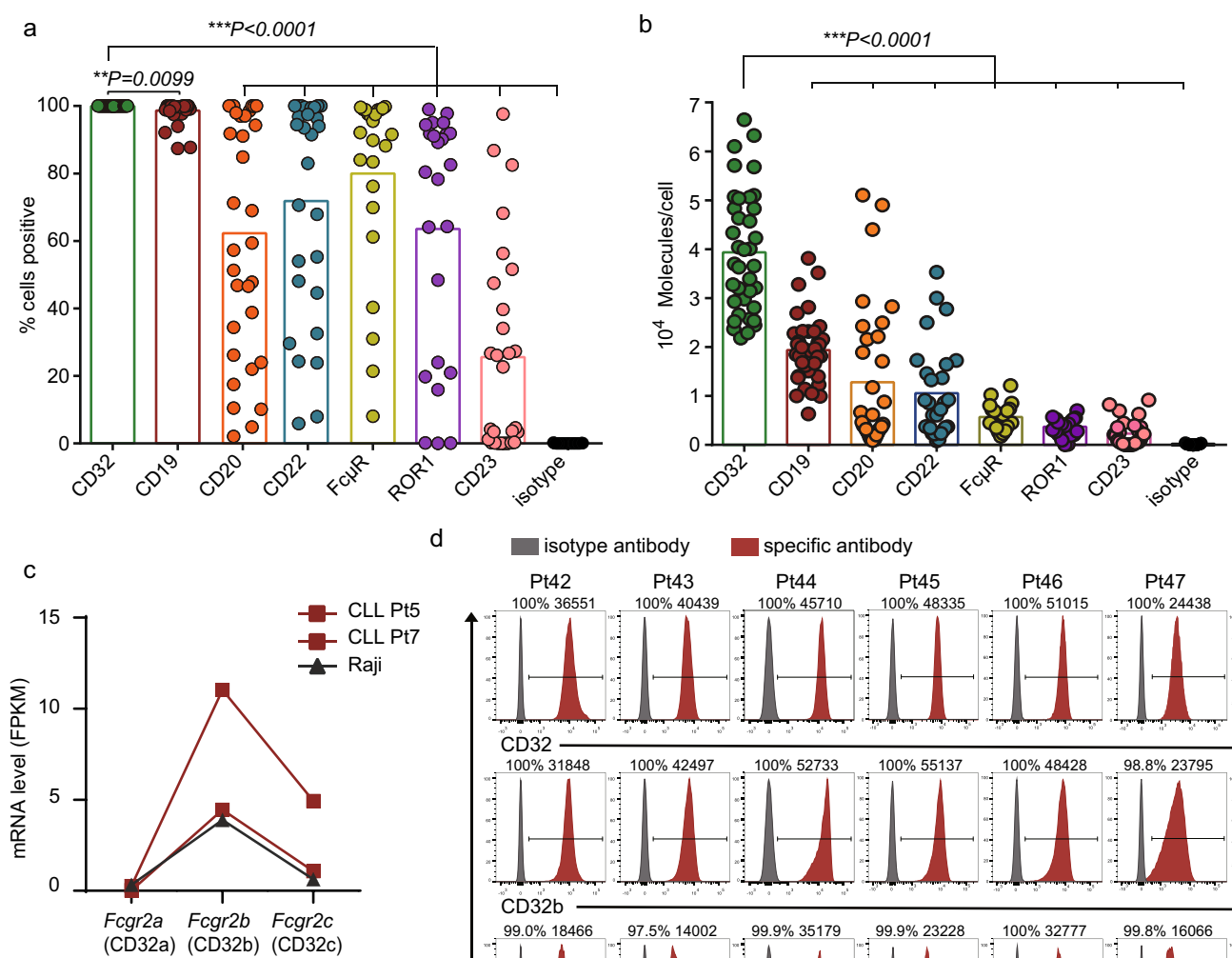

d isotype antibody
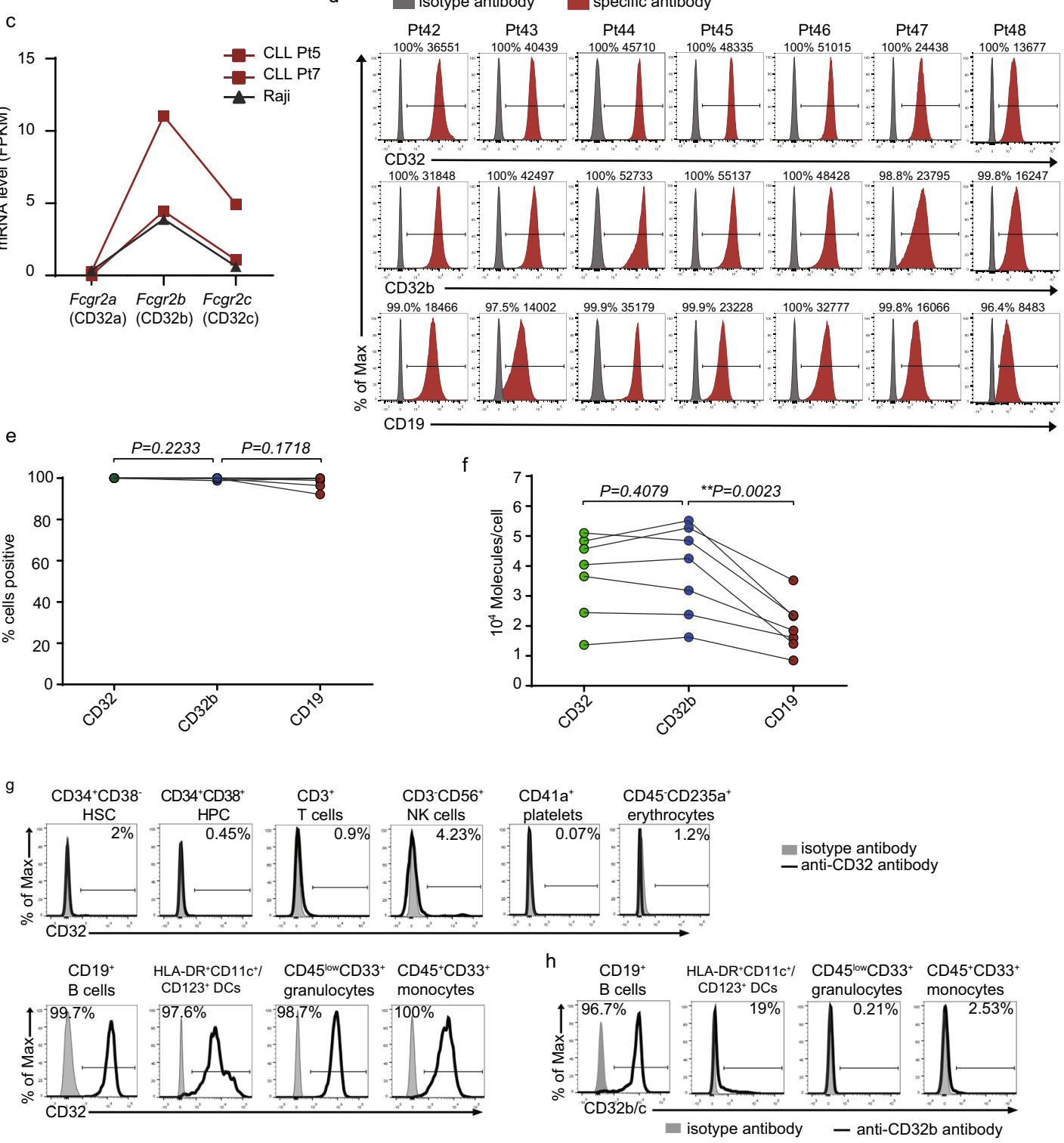

Fig. 1 (See legend on previous page.) 


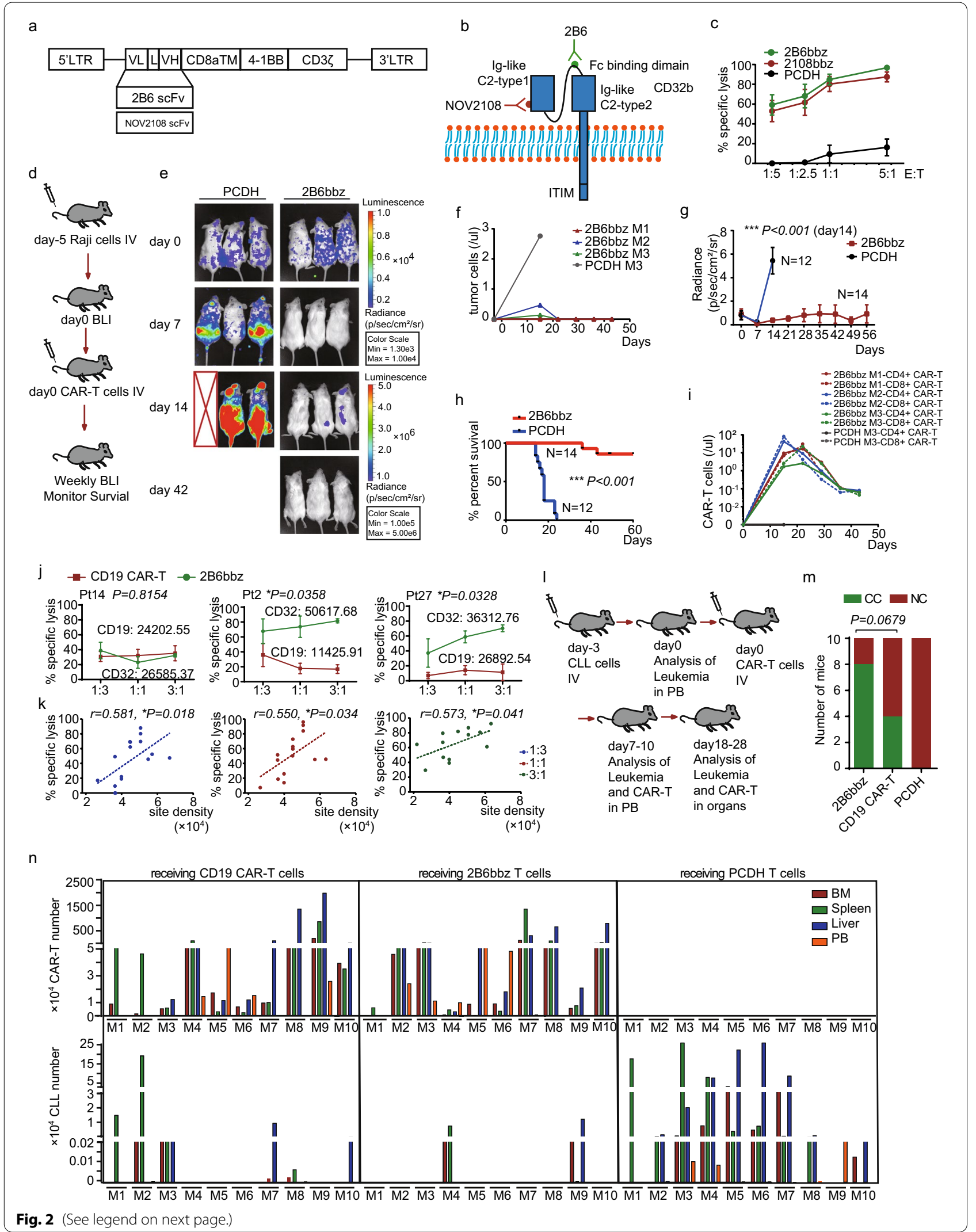




\section{(See figure on previous page.)}

Fig. 2 CD32b CAR-T efficacy against Raji cells and primary CLL cells. a Diagram indicating constructions of two CD32b CAR sequences (scFvs from clone 2 B6 or NOV2108). b NOV2018 scFv binds Ig-like C2-type 1 domain of CD32b, whereas 2B6 binds binding domain of CD32b. c Cytotoxicity of CD32b CAR-T targeting Raji cells after incubation for $36 \mathrm{~h}$ at the indicated effector-to-target (E:T) ratios; control T cells were used as negative controls. d Schematic of the Raji xenograft model. NSG mice were injected via tail vein with $3 \times 10^{5}$ luciferase ${ }^{+}$Raji cells on day-5. Bioluminescent imaging was performed on day 0 to quantify engraftment and then weekly measured. Control T cells or 2 B6bbz T cells $\left(1 \times 10^{6}\right)$ were injected IV on day 0. e Representative bioluminescent imaging at day $0,7,14$ and 42 after injection of Raji cells. f Flow cytometric analysis of Raji cells in peripheral blood from Raji-NSG mice (from e). $\mathbf{g}$ Bioluminescent signal for each treatment group over time. Data represent mean values of each group \pm SD. $\mathbf{h}$ Log-rank survival curve was used for survival analysis of Raji xenograft mice treated by $2 \mathrm{~B} 6 \mathrm{bbz}$ or control $\mathrm{T}$ cells. Data of $\mathrm{g}$ and $\mathrm{h}$ were summarized from 4 independent experiments. (Control, $n=12 ; 2 B 6 b b z, n=14$ ). i Flow cytometric analysis of CAR-T cells in peripheral blood from Raji-NSG mice (from e). $\mathbf{j}$ Specific cytotoxicity targeting of CLL by 2B6bbz and CD19 CAR-T cells after incubation with primary CLL cells for $36 \mathrm{~h}$ at the indicated E:T ratios; Three representative CLL patient examples are shown. $\mathbf{k}$ Correlation between 2B6bbz T cytotoxicity and CD32 density across different patient CLL samples. I Schematic of the primary CLL xenograft model. NSG mice were sublethally irradiated (150 cGy) on day -3 and injected with $2-4 \times 10^{7} \mathrm{CLL}$ PBMCs via the tail vein on day -3 . Engraftment was confirmed by flow cytometry in PB around day 0 . Mice were then injected with $5 \times 10^{5} 2 \mathrm{~B} 6 \mathrm{bbz}$ T, CD19 CAR-T cells or control T cells via the tail vein and bled weekly to quantify CLL burden. $\mathbf{m}$ Response of primary CLL-NSG mice treated with 2B6bbz T (CC, $n=8 ; N C, n=2), C D 19$ CAR-T $(C C, n=4 ; N C, n=6)$ or control T cells $(N C, n=10)$. $\mathbf{n}$ Number of CAR-T and tumor residue in PB, BM, liver and spleen from CLL-NSG mice after receiving CAR-T cells for 18 days. Data of $m$ and $n$ were summarized from four independent experiments. M indicates mouse. CC, complete clearance (defined as tumor residual less than $0.001 \%$ in all the tissues detected); NC, not clearance (mouse couldn't be defined as CC); BM, bone marrow; PB, peripheral blood. Chi-square test was used for statistical analysis in m. Log-rank (Mantel-Cox) test was used for statistical analysis in h. Unpaired two-tailed Student's t test was used for statistical analyses in $\mathrm{g}$ and $\mathrm{j}$. Pearson correlation analysis was used in $\left.\mathrm{k} .{ }^{*} P<0.05,{ }^{* *} P<0.01,{ }^{* * *} P<0.001\right)$

\section{Abbreviations}

CAR: Chimeric antigen receptor; CLL: Chronic lymphocytic leukemia; CR: Complete response; r/r: Refractory and relapsed; FcyRllb: Low affinity immunoglobulin gamma Fc region receptor II-b; mAb: Monoclonal antibodies; scFv: Single-chain variable fragment; FPKM: Expected number of Fragments Per Kilobase of transcript sequence per Millions base pairs sequenced; PBMC Peripheral blood mononuclear cell; PB: Peripheral blood; CC: Complete clearance; NC: Not clearance; HSC: Hematopoietic stem cell; HPC: Hematopoietic progenitor cell; BM: Bone marrow; NK: Natural killer; DC: Dendritic cells.

\section{Supplementary Information}

The online version contains supplementary material available at https://doi. org/10.1186/s13045-021-01160-9.

Additional file 1: Table S1. Patients'information and expressional characteristics of all antigens.

Additional file 2. Materials and Methods.

Additional file 3: Fig. S1. CD32 expresses higher than other antigens on primary CLL samples. a Flow cytometric analysis of surface expression of CD32, CD19, CD20, CD22, ROR1, FchR and CD23 in 4 CLL samples. CLL cells were gated as $\mathrm{CD} 19^{+} \mathrm{CD}^{+}$cells. $\mathbf{b}$ Site density comparison between CD32 and CD19 in CLL patients $(n=41)$. c Quantification of mRNA transcripts from Raji cells, THP-1 cells and leukemic cells of 2 CLL patients by RNA sequencing. $\mathbf{d} 293 \mathrm{~T}$ cells were genetically modified to express CD32a and CD32b; cells (unmodified 293T, CD32a ${ }^{+} 293$ T and CD32b ${ }^{+}$293T) were stained with 2B6-scFv-Flag Ab, 2108-scFv-Flag Ab and anti-CD32 mAb (clone FUN-2). Fig. S2. CD32b CAR-T against Raji cells in vitro and in vivo. a Flow cytometry analysis of CAR expression in T cells following lentiviral transduction. Left, control T cells; middle, T cells transduced with 2B6bbz; right, $T$ cells transduced with 2108bbz. CARs were detected by CD32b-His followed by an anti-His-APC second antibody stain. b Flow cytometric analysis of surface expression of CD32b on the B-cell leukemia cell lines Mec-1 and Raji. c Antigen-specific cytokine production in response to $\mathrm{CD} 32 \mathrm{~b}^{+}$Raji cells. 2B6bbz and control T cells were incubated with Raji cells $\left(2 \times 10^{4}\right)$ respectively for $24 \mathrm{~h}$ in E:T ratio of 1:1. The various proteins in the culture supernatant were detected using the bead-based "LEGENDplex multi-analyte assay." d Representative flow cytometric plot and flow gating strategy of peripheral blood from Raji-NSG mice 15 days after receiving 2B6bbz or control T cells. Fig. S3. CD32b CAR-T had potent cytotoxicity to primary CLL. a Flow cytometry analysis of CAR expression in T cells following lentiviral transduction. Left, control T cells; left-center, T cells transduced with 2B6bbz; right-center, T cells transduced with 2108bbz; right, T cells transduced with CD19 CAR. CARs were detected by CD32bHis/CD19-Fc followed by an anti-His-APC/anti-Fc second antibody stain. $\mathbf{b}$ Specific cytotoxicity of 2B6bbz, 2108bbz or control T cells after coculture with primary CLL cells for $36 \mathrm{~h}$ at the indicated E:T ratios; c Antigen-specific cytokine production of 2B6bbz, CD19 CAR-T and control T cells in response to $24 \mathrm{~h}$ co-culturing with primary CLL cells. Fig. S4. 2 B6bbz T against primary CLL cells in vivo. a Flow cytometric analysis of tumor percentage in peripheral blood before T cells infusion. b Representative flow cytometry plot of bone marrow, spleen and peripheral blood, from CLL-NSG mice after receiving CAR-T cells for 18 days. c Treatment response of 2B6bbz and CD19 CAR-T cells against mice transplanted with different antigen density primary CLL cells. $\mathbf{d}$ Flow cytometric analysis of CD32 and CD19 expression on CLL cells from peripheral blood of NSG mice before and at 7 days after CAR-T infusion. e Quantification of the percentage of CLL cells in peripheral blood, bone marrow, spleen and liver from CC and NC CLL-NSG mice after receiving CAR-T cells for 18 days. $\mathbf{f}$ Number of CAR-T cells in peripheral blood, bone marrow, spleen and liver from CC and NC CLL-NSG mice after receiving CAR-T cells for 18 days. Fig. S5. mRNA expression profile of $f c g r 2 b$ and fcgr2c in normal human tissues at mRNA level according to publicly available database (BioGPS: fcgr2b, http://biogps.org/\#goto=genereport\& id=2213; fcgr2c, http://biogps.org/\#goto=genereport\&id=9103)

\section{Acknowledgements}

We thank Xiaolei Pei, Rui Chen and Chunxiao Zhao (Institute of Hematology, Chinese Academy of Medical Sciences) for assisting some laboratory experiments. We thank members of our team for critical discussion and suggestions.

\section{Authors' contributions}

GW, XS and XF designed the study; GW and XS performed most of the experiments and analyzed the data; $S Z, C L, Y M$ and $M L$ helped with the plasmid construction and animal experiments; $\mathrm{QN}, Z \mathrm{ZF}, \mathrm{XY}, \mathrm{YJ}$ and $\mathrm{DZ}$ helped with the basic lentivirus and plasmid and CAR-T generation system; YX, SW, HC, HH and EJ helped with the collection of patient samples; JP and QD helped with the study design and provided clinical consultation; XS, GW and XF wrote the manuscript; QD, JP and XF jointly directed and supervised the study. All authors read and approved the final manuscript.

\section{Funding}

This work was supported by the National Key R\&D Program of China (2019YFA0110200), the Non-profit Central Research Institute Fund of Chinese Academy of Medical Sciences (2019-RC-HL-013), the National Natural Science 
Foundation of China $(81870090,82000191)$ and the Tianjin Science Funds for Distinguished Young Scholars (17JCJQJC45800).

\section{Availability of data and materials}

All data needed to evaluate the conclusions in the paper are present in the paper or the additional files.

\section{Declarations}

\section{Ethics approval and consent to participate}

The studies were conducted in accordance with the Declaration of Helsinki and approved by the Ethics Committee of the State Key Laboratory of Experimental Hematology, Institute of Hematology and Hospital of Blood Disease, Chinese Academy of Medical Sciences and Peking Union Medical College (approval number: KT2020005-EC-3).

\section{Consent for publication}

Not applicable.

\section{Competing interests}

The authors declare that they have no competing interests.

\section{Author details}

${ }^{1}$ State Key Laboratory of Experimental Hematology, National Clinical Research Center for Blood Diseases, Institute of Hematology and Blood Diseases Hospital, Chinese Academy of Medical Sciences and Peking Union Medical College, Tianjin 300020, China. ${ }^{2}$ Key Laboratory of Immune Microenvironment and Disease of the Ministry of Education, Tianjin Medical University, Tianjin 300070, China. ${ }^{3}$ Institut Pasteur of Shanghai, Chinese Academy of Sciences, Shanghai 200031, China. ${ }^{4}$ Department of Hematology, Tianjin First Central Hospital, School of Medicine, Nankai University, Tianjin 300192, China. ${ }^{5}$ Department of Thoracic Surgery, Hubei Cancer Hospital, Tongji Medical College of Huazhong University of Science and Technology, Wuhan 430079, China. ${ }^{6}$ Department of Breast Surgery, The First Affiliated Hospital, School of Medicine, Zhejiang University, Hangzhou 310000, China. ${ }^{7}$ Central Laboratory, Fujian Medical University Union Hospital, Fuzhou 350001, China. ${ }^{8}$ Department of Pathogen Biology, School of Basic Medical Sciences, Tianjin Medical University, Tianjin 300070, China. ${ }^{9}$ State Key Laboratory of Experimental Hematology, Boren Clinical Translational Center, Department of Hematology, Beijing Boren Hospital, Beijing 100070, China.

Received: 31 May 2021 Accepted: 31 Auqust 2021

Published online: 16 September 2021

\section{References}

1. Barr PM, Munir T, Brown JR, O'Brien SM, Barrientos JC, Reddy NM, et al. Final analysis from RESONATE: six-year follow-up in patients (pts) with previously treated chronic lymphocytic leukemia or small lymphocytic lymphoma (CLL/SLL) on ibrutinib. J Clin Oncol. 2019; 37(15).

2. Stilgenbauer S, Schnaiter A, Paschka P, Zenz T, Rossi M, Dohner K, et al. Gene mutations and treatment outcome in chronic lymphocytic leukemia: results from the CLL8 trial. Blood. 2014;123(21):3247-54.

3. Siddiqi T, Soumerai JD, Dorritie KA, Stephens DM, Riedell PA, Arnason $\mathrm{JE}$, et al. Updated follow-up of patients with relapsed/refractory chronic lymphocytic leukemia/small lymphocytic lymphoma treated with lisocabtagene maraleucel in the phase 1 monotherapy cohort of transcend CLL 004, including high-risk and ibrutinib-treated patients. Blood. 2020;136(Supplement 1):40-1.

4. Frey NV, Gill S, Hexner EO, Schuster S, Nasta S, Loren A, et al. Long-term outcomes from a randomized dose optimization study of chimeric antigen receptor modified T cells in relapsed chronic lymphocytic leukemia. $J$ Clin Oncol. 2020;38(25):2862-71.

5. Ramakrishna S, Highfill SL, Walsh Z, Nguyen SM, Lei H, Shern JF, et al. Modulation of target antigen density improves CART-cell functionality and persistence. Clin Cancer Res. 2019;25(17):5329-41.

6. Bruhns P, lannascoli B, England P, Mancardi DA, Fernandez N, Jorieux S, et al. Specificity and affinity of human Fcy receptors and their polymorphic variants for human IgG subclasses. Blood. 2009;113(16):3716-25.

7. Herman SEM, Montraveta A, Niemann CU, Mora-Jensen H, Gulrajani M, Krantz F, et al. The Bruton Tyrosine Kinase (BTK) inhibitor acalabrutinib demonstrates potent on-target effects and efficacy in two mouse models of chronic lymphocytic leukemia. Clin Cancer Res. 2017;23(11):2831-41.

8. Xia YC, Schuliga M, Shepherd M, Powell M, Harris T, Langenbach SY, et al. Functional expression of $\mathrm{IgG}-\mathrm{Fc}$ receptors in human airway smooth muscle cells. Am J Respir Cell Mol Biol. 2011;44(5):665-72.

9. Bruggeman CW, Houtzager J, Dierdorp B, Kers J, Pals ST, Lutter R, et al. Tissue-specific expression of $\mathrm{lg} \mathrm{G}$ receptors by human macrophages ex vivo. PLoS ONE. 2019;14(10):e0223264.

10. Drent E, Themeli M, Poels R, de Jong-Korlaar R, Yuan H, de Bruijn J, et al. A rational strategy for reducing on-target off-tumor effects of CD38-chimeric antigen receptors by affinity optimization. Mol Ther. 2017;25(8):1946-58.

11. Morsut L, Roybal KT, Xiong X, Gordley RM, Coyle SM, Thomson M, et al. Engineering customized cell sensing and response behaviors using synthetic notch receptors. Cell. 2016;164(4):780-91.

12. Cho JH, Collins JJ, Wong WW. Universal chimeric antigen receptors for multiplexed and logical control of T cell responses. Cell. 2018;173(6):14261438.e11.

\section{Publisher's Note}

Springer Nature remains neutral with regard to jurisdictional claims in published maps and institutional affiliations.
Ready to submit your research? Choose BMC and benefit from:

- fast, convenient online submission

- thorough peer review by experienced researchers in your field

- rapid publication on acceptance

- support for research data, including large and complex data types

- gold Open Access which fosters wider collaboration and increased citations

- maximum visibility for your research: over $100 \mathrm{M}$ website views per year

At BMC, research is always in progress.

Learn more biomedcentral.com/submissions 